\title{
Implicación de la investigación de resultados en salud en la mejora continua de la calidad asistencial del Sistema Nacional de Salud
}

Soto Álvarez J. Implicación de la investigación de resultados en salud en la mejora continua de la calidad asistencial del Sistema Nacional de Salud. An Med Interna (Madrid) 2007; 24: 517-519.

\section{INTRODUCCIÓN}

La evaluación de la calidad asistencial del Sistema Nacional de Salud (SNS) de nuestro país es cada vez más importante de cara a renovar el conocimiento científico, mejorar en las prácticas sanitarias, actualizar el aprendizaje de los profesionales sanitarios y conseguir los mejores resultados y los mayores progresos en la salud de los ciudadanos (1).

Los principales objetivos de la calidad asistencial van a ser: a) prestar asistencia sanitaria acorde al estado de la ciencia y a las evidencias existentes; b) conseguir una atención sanitaria que satisfaga al paciente; c) asegurar la accesibilidad y continuidad de los cuidados en los pacientes; d) emplear intervenciones sanitarias apropiadas según las necesidades de los pacientes y acorde a la patología que padecen; e) lograr los mejores resultados en salud en los ciudadanos; y f) conseguir el máximo de beneficio en salud con el mínimo consumo de recursos (2).

Con el fin de conseguir una calidad asistencial de alto nivel, será necesario prestar una atención sanitaria focalizada en seguridad (evitar morbilidad en los pacientes), que sea efectiva (asistencia basada en la mejor evidencia científica existente, intentando evitar el sobreuso o la infrautilización de los recursos disponibles), que este centrada en el paciente (respetando sus valoraciones y opiniones subjetivas), que se emplee en el momento apropiado (evitar retrasos innecesarios para el paciente), que sea eficiente (consiguiendo los mejores resultados con el menor consumo de recursos posibles) y que sea equitativa (provea los mismos cuidados sanitarios para las mismas enfermedades, sin distinción social o de ubicación del paciente (3).

Por lo tanto, la manera en la que se van a valorar, cuantificar y difundir los resultados en salud (cambios en el estado de salud actual o futuro, en cualquiera de las variables y/o parámetros que la definen, que pueden ser atribuidos a la asistencia sanitaria recibida) va a ser un aspecto clave a la hora de poder evaluar la calidad asistencial ofrecida, siempre que se cumplan las siguientes premisas: a) deberían identificar resultados específicos de cada enfermedad o adaptados a la enfermedad que se evalúa; b) pueden evaluarse una o varias dimen- siones de la salud; y c) los resultados obtenidos deberían ser validos, fiables y relevantes para los profesionales sanitarios y la sociedad $(4,5)$.

La investigación de resultados en salud (IRS) es una disciplina orientada y focalizada a cuantificar, analizar e interpretar los resultados en salud que generan las distintas intervenciones sanitarias (medicamentos, procedimientos quirúrgicos, técnicas diagnosticas, productos sanitarios, etc.) en condiciones de práctica médica habitual, lo que va a ayudar tremendamente al profesional sanitario y a otros agentes decisores a tomar decisiones más acertadas y razonables, ya que van a ser capaces de saber cómo se comportan las intervenciones evaluadas en condiciones de uso rutinario del mundo real (6-8).

Esta nueva área de conocimiento se va a focalizar en valorar distintos resultados en salud tras la aplicación de diferentes intervenciones sanitarias, tales como resultados clínicos, económicos, humanísticos y de gestión sanitaria (9).

En relación a los resultados clínicos, el área de mayor interés radica en intentar averiguar los beneficios terapéuticos de las intervenciones en condiciones de uso habituales, esto es, conocer su grado de efectividad clínica. Otros datos de interés son conocer la evolución de los síntomas de las enfermedades, disponer de datos de morbilidad (reingresos, complicaciones, recidivas, exacerbaciones, etc.) y mortalidad de las enfermedades a medio-largo plazo, poder diseñar herramientas de cribaje para poder diagnosticar éstas rápidamente y conocer el nivel de cumplimiento terapéutico y el grado de persistencia de los pacientes con las alternativas terapéuticas prescritas por el médico (10).

Sobre los resultados económicos, esta disciplina está muy orientada a conocer la eficiencia de las intervenciones sanitarias empleadas (relación entre los resultados clínicos obtenidos y los costes necesarios para su consecución) en la práctica rutinaria y las posibles ventajas económicas (ahorro de recursos) derivadas de su utilización habitual, efectuando análisis económicos, los cuáles podrán ser de diferentes tipos (costebeneficio, coste-efectividad, coste-utilidad, de minimización de costes o coste-consecuencia) según se valoren y midan los resultados clínicos encontrados. En los últimos años, están tomando mucha relevancia para los decisores otro tipo de 
datos, como son el consumo de recursos derivado del manejo de las enfermedades (estudios de coste de las enfermedad) y cómo afecta al presupuesto existente la comercialización de una nueva opción terapéutica (análisis del impacto presupuestario), por lo que es de esperar que cada vez se realicen más análisis de estas características (11).

En lo referente a los resultados humanísticos, la IRS se centra en proporcionar información de resultados percibidos y comunicados por los pacientes como indicadores únicos del impacto de la enfermedad y de la efectividad lograda con la intervención sanitaria aplicada, desde una visión puramente subjetiva del paciente. Por lo tanto, la IRS va a evaluar cómo las distintas opciones terapéuticas van a afectar a la calidad de vida y el nivel de satisfacción de los pacientes, y a estudiar el estado de salud y el grado de preferencias de los pacientes sobre las alternativas terapéuticas administradas, junto con la valoración de la discapacidad y el estado funcional que produce la enfermedad y su tratamiento en el paciente (12-14).

Por último, en relación con la gestión sanitaria, esta disciplina se centra en evaluar los servicios sanitarios del sistema nacional de salud, así como en la elaboración y conocimiento de indicadores sanitarios que puedan reflejar los resultados en salud existentes en la población, tales como la tasa de infartos de miocardios o de ictus anuales en una determinada región o en una Comunidad Autónoma, ya que su conocimiento y evaluación va a servir para conocer sí las intervenciones sanitarias empleadas son las más adecuadas, o sí habría que replantearse las políticas sanitarias a ese nivel. De manera complementaria, la IRS va a valorar si existe un uso apropiado de los recursos (adecuación e idoneidad), en un intento por incrementar la eficiencia y equidad $(15,16)$.

Para poder efectuar estudios que permitan disponer de todos estos resultados, la IRS emplea diseños metodológicos validos y precisos derivados de la investigación clínica (valoración de efectos clínicos, evaluación de la morbi-mortalidad de los pacientes, conocimiento del grado de cumplimiento, etc.), la epidemiología clínica (incidencia y prevalencia de la enfermedad, valoración de los factores de riesgo de distintas patologías, indicadores sanitarios poblacionales, etc.), la economía de la salud (evaluación de los costes, análisis del impacto presupuestario, evaluaciones económicas, etc.) y la psicología y psicometría (valoración de cómo afecta la enfermedad y el tratamiento administrado a la calidad de vida y el nivel de satisfacción de los pacientes) (17-19). De esta manera, los resultados obtenidos van a ser de gran rigor científico y amplia relevancia para los decisores y clínicos a la hora de tomar decisiones clínicas y de política sanitaria.

Al final, esta disciplina se va a nutrir de diferentes fuentes y diseños metodológicos a la hora de elaborar sus estudios y análisis, en unos casos serán fuentes primarias (ensayos clínicos, diseños observacionales, estudios epidemiológicos, análisis farmacoeconómicos, etc.), mientras que en otros casos tendrá que recurrir a fuentes secundarias (meta-análisis, revisiones sistemáticas, revisiones de seguridad, estudios de coste de la enfermedad, etc.), lo que va a enriquecer la información final disponible para el clínico y el decisor $(20,21)$.

Los estudios de IRS van a mostrar el verdadero valor aportado por las intervenciones sanitarias empleadas para tratar distintas patologías, una vez éstas se empiecen a utilizar en condiciones de uso habitual, acorde a la práctica médica diaria $\mathrm{y}$, por lo tanto, van a ser de gran ayuda a la hora de diseñar estrategias y políticas orientadas a conseguir los mejores resultados y beneficios clínicos en el paciente y mejorar la eficiencia económica del SNS (22).

En este momento, dentro del SNS es necesario evolucionar desde la gestión del gasto a un nuevo paradigma, como es la gestión de la calidad. La calidad asistencial va a representar el grado en el que la atención sanitaria dada a un individuo o a una población desde el SNS es capaz de maximizar la ganancia en salud de la población. Para su consecución, va a ser necesario disponer de excelentes sistemas de información, protocolizar todos los procesos, tanto de atención primaria como de asistencia hospitalaria, integrar toda la información generada en los centros sanitarios y cuantificar y medir los resultados en salud conseguidos. Además, será recomendable valorar si éstos son consistentes y consecuentes con las evidencias científicas disponibles y los recursos que la sociedad ha decidido destinar al cuidado sanitario y, por lo tanto, si la atención sanitaria ofrecida es coste-efectiva.

La calidad asistencial es, hoy por hoy, el cuarto pilar de la atención sanitaria junto con la sostenibilidad, la eficiencia y la equidad, y su mejora e incremento permitiría poder racionalizar (e incluso, en algunos casos reducir) los costes sanitarios del SNS. La búsqueda y consecución de una elevada calidad asistencial debería ser una estrategia política global y unificada de todos los servicios de salud autonómicos y del propio Ministerio de Sanidad, además de ser una exigencia moral de todos agentes integrantes del SNS (profesionales, gestores, industria farmacéutica, etc.).

De hecho, en la ley de cohesión y calidad del sistema nacional de salud ya se establece la creación de una agencia de calidad cuyo fin último es lograr un incremento en el nivel de calidad asistencial del SNS, al considerarse como un asunto totalmente estratégico (23).

En este contexto, sería deseable que a la hora de evaluar la calidad asistencial dentro del SNS a través de criterios, indicadores y estándares, se empezase a incluir una medición exhaustiva de los resultados en salud conseguida y se relacionasen de manera directa con el gasto sanitario necesario para su consecución. Por otra parte, sería recomendable que se creasen equipos de expertos en investigación de resultados en salud que aconsejasen a las autoridades sanitarias y a otros decisores sobre cuándo y cómo emprender estos estudios de IRS.

El proceso de reorientación y transformación del SNS a corto-medio plazo debería asentarse en el incremento de la calidad asistencial en términos de ganancia en resultados en salud, en implicar más al ciudadano y a la sociedad en general, en buscar la eficiencia económica y la propia viabilidad del sistema y en desarrollar políticas y estrategias coherentes para promover la salud y evitar la aparición de enfermedades con un alto coste de tratamiento y de graves consecuencias en merma de productividad para la sociedad. En este proceso de búsqueda, los estudios de IRS van a ser unos aliados estratégicos que nos van a proporcionar evidencias y nos van a ayudar a priorizar las intervenciones sanitarias a implantar, además de servir de soporte para dirigir adecuadamente los esfuerzos para poder lograr la mayor rentabilidad social de nuestro SNS.

\section{J. SOTO ÁLVAREZ}

Departamento de Investigación de Resultados en Salud y Farmacoeconomía. Unidad Médica. Pfizer España 


\section{Bibliografía}

1. Aranaz JM. La calidad asistencial: Una necesidad en la práctica clínica. En: Matías-Guiu J, Lainez JM. Gestión sanitaria y asistencia neurológica. JR Proas Editores. Barcelona, 1994.

2. Ruiz López P. La medición de la calidad asistencial. Rev Clin Esp 2001; 201: 561-2.

3. Rodríguez Pérez MP. Calidad asistencial: Conceptos, dimensiones y desarrollo operativo. En: Gestión clínica: Desarrollo e instrumentos. Ed Luis Angel Oteo. Edición Díaz de Santos, 2006.

4. Donabenian A. The role of outcomes in quality assessment and assurance. Qual Rev Bull 1992; 18: 356-60.

5. Peiro S, Lorenzo S. La difusión a los ciudadanos de los resultados de la asistencia sanitaria. Rev Calidad Asistencial 2000; 15: 391-3.

6. Guadagnoli E, McNeil B. Outcomes research: Hope for the future or the latest rage? Inquiry 1994; 31: 14-24.

7. Weeks J, Pfister DG. Outcomes research studies. Oncology 1996; 10 (Supl. 11): 29-34

8. Clancy CM, Eisenberg JM. Outcomes research: Measuring the end results of health care. Science 1998; 282: 245-6.

9. Rapier CM. An introduction to outcomes research. Brookwood Medical Publication, 1996.

10. Freud D, Lave J, Clancy C, Hawker G, Hasselblad V, Kaller R, et al. Patient outcomes research teams: Contribution to outcomes and effectiveness research. Annv Rev Public Health 1999; 20: 337-59.

11. Lancry PJ, Oconnor R, Stempel D, Raz M, et al. Using health outcomes data to inform decision-making healthcare payer perspective. Pharmacoeconomics 2001; 19 (Supl. 2): 39-47.

12. Rodríguez Artalejo F. Investigación de resultados (outcomes research) en el área de envejecimiento. Rev Esp Geriatr Gerontol 2001; 36: 20-3.
13. Ruiz de Velasco I, Quintana JM, Padierne JA, Arostegui I, Bernal A, Perez-Izquierdo J, et al. Validez del cuestionario de calidad de vida SF36 como indicador de resultados de procedimientos médicos y quirúrgicos. Rev Calidad Asistencial 2002; 17: 206-12.

14. Díaz R. Satisfacción del paciente: principal motor y centro de los servicios sanitarios. Rev Calidad Asistencial 2002; 17: 22-29.

15. Carrasco G, Pallarés A, Cabre L. Costes de la calidad en medicina intensiva. Guía para gestores clínicos. Rev Calidad Asistencial 2005; 20: 271-84.

16. Hernandez S, Garcia J, Jiménez C, Escuin F, Mahillo B, Herruzo R, et al. Resultados e impacto de una vía clínica para trasplante renal tras un año de desarrollo. Rev Calidad Asistencial 2003; 18: 9-19.

17. Petitti DB. Epidemiologic issues in Outcomes Research. En: Brownson RC, Petitti DB. Applied epidemiology theory to practice. New York. Oxford University Press ; 1998 : 249-75

18. Bruner DW, Mousas B, Konski A, Roach M, Bondy M, Scarintino C, et al. Outcomes research in cancer clinical trial cooperative groups: the RTOG model. Qual Life Res 2004; 13: 1025-41.

19. Moliner J. El uso del método epidemiológico para la evaluación de la calidad de la asistencia (I). Rev Calidad Asistencial 2002; 17: 292-304.

20. Mitchell JB. Using Medicare claims for outcomes research. Med Care 1994; 32 (7 Suppl): JS38-JS51.

21. Canto JG, Kiefe CI, Williams D, et al. Comparison of outcomes research with clinical trials using pre-existing data. Am J Cardiol 1999; 84: 923-7.

22. Epstein RS, Sherwood LM. From outcomes research to disease management: a guide for the perplexed. Ann Intern Med 1996; 124: 832-7.

23. Ley $16 / 2003$, de 28 de mayo, de cohesión y calidad del sistema nacional de salud. BOE del 29 de mayo de 2003. 